Analysis of Faraday rotation in JET polarimetric measurements

This article has been downloaded from IOPscience. Please scroll down to see the full text article.

2011 Plasma Phys. Control. Fusion 53035001

(http://iopscience.iop.org/0741-3335/53/3/035001)

View the table of contents for this issue, or go to the journal homepage for more

Download details:

IP Address: 160.80.89.21

The article was downloaded on 28/01/2011 at 09:58

Please note that terms and conditions apply. 


\title{
Analysis of Faraday rotation in JET polarimetric measurements
}

\author{
F P Orsitto ${ }^{1}$, A Boboc ${ }^{2}$, P Gaudio ${ }^{3}$, M Gelfusa ${ }^{3}$, E Giovannozzi ${ }^{1}$, \\ C Mazzotta $^{1}$, A Murari ${ }^{4}$ and JET EFDA Contributors ${ }^{5}$ \\ JET-EFDA, Culham Science Centre, OX14 3DB, Abingdon, UK \\ ${ }^{1}$ Associazione EURATOM-ENEA C R Frascati 00044 Frascati, Italy \\ ${ }^{2}$ Association EURATOM-CCFE Culham Science Centre Abingdon OX14 3DB, UK \\ 3 Associazione EURATOM-ENEA Università Roma II Tor Vergata, Italy \\ ${ }^{4}$ Associazione EURATOM-ENEA Consorzio RFX Padova, Italy \\ E-mail: orsitto@frascati.enea.it
}

Received 29 April 2010, in final form 8 November 2010

Published 27 January 2011

Online at stacks.iop.org/PPCF/53/035001

\begin{abstract}
The paper deals with JET polarimeter measurements and in particular it presents a study of the Faraday rotation angle, which is used as a constraint in equilibrium codes. This angle can be calculated by means of the rigorous numerical solution of Stokes equations. A detailed comparison of calculations is carried out with the time traces of measurements, inside a limited dataset representative of JET discharges: in general, it is found that the Faraday rotation angle and Cotton-Mouton phase shift measurements can be represented by the numerical solution to Stokes equations. To obtain this agreement in particular for Faraday rotation, a shift of the magnetic surfaces must be included. This results in an improvement of the position of the magnetic surfaces as calculated by the EFIT equilibrium code. The approximated linear models normally used can be applied only at low density and current. The Cotton-Mouton is calculated at high plasma density including the contribution by the Faraday rotation angle. For high plasma current the non-linear terms in the propagation equations can be important. These conclusions have some impact on the mathematical form of the polarimetric constraints (Faraday and Cotton-Mouton) in equilibrium codes.

(Some figures in this article are in colour only in the electronic version)
\end{abstract}

\section{Introduction}

The measurements of polarimetry in tokamak plasmas can give important information on plasma current and density [1]. In a plasma, in the presence of a magnetic field, the polarization

5 See the appendix of Romanelli $\mathrm{F}$ et al 2008 Proc. 22nd IAEA Int. Conf. on Fusion Energy Conference 2008 (Geneva, Switzerland). 
plane of a laser beam propagating along the magnetic field rotates (the Faraday effect). Whereas, if the laser beam is propagating perpendicular to the magnetic field there is a change in the ellipticity of the polarization (the Cotton-Mouton effect). In a beam propagating vertically along a line of sight in a poloidal plane of a tokamak, both effects are present: due to the topology of the magnetic field in a tokamak there is a component of the magnetic field along the toroidal axis (which is perpendicular to the beam) and a component of the poloidal magnetic field (generated by the plasma current) along the propagation line. The polarization of the beam becomes elliptical because the plasma is birefringent, i.e. the optical properties of the plasma are described by a dielectric tensor instead of a simple dielectric scalar. As a first approximation, it is possible to consider the two effects as being independent: specifically, the Faraday effect depending only on the magnetic field parallel to the beam direction times the plasma density, while the Cotton-Mouton depends only on the plasma density and the perpendicular (to the line of sight) magnetic field squared. For example, in low density plasmas this scheme is valid (see section 2).

Since the structure of the propagation equations of polarization inside the plasma in the magnetic field of a tokamak couples the Faraday and Cotton-Mouton (see section 2 and 5), the two effects must be taken into account at the same time, in a rigorous approach to calculate the change in polarization of a laser beam.

In a previous paper [2], the analysis of Cotton-Mouton measurements was carried out and the consistency of measurements with the Stokes equation models was assessed. It was shown that the Cotton-Mouton phase shift angle can be calculated by means of the rigorous solution of Stokes equations, which define the spatial evolution of the polarization of the laser beam inside the plasma. The coupling between Faraday and Cotton-Mouton was demonstrated as being important for large Faraday effects. In fact, to analyse the coupling between Faraday and Cotton-Mouton a new approximate analytic solution (type II) [2] was introduced. Using the topology of tokamak magnetic fields, an ordering was found between the components of the vector $\vec{\Omega}$ appearing in the Stokes equations (see section 2), leading to a simplified analytic solution, which exhibits (i) a sensible dependence of the Cotton-Mouton phase shift upon the Faraday rotation angle, while (ii) the Faraday rotation is not dependent on Cotton-Mouton (see comments to (2.12)).

In this paper the analysis of Faraday rotation measurements is carried out, following the same method developed in [2], using the Stokes equations and their numerical and approximate solutions. The coupling between Faraday and Cotton-Mouton is further analysed, from the general point of view, using a new set of non-linear equations derived from the Stokes ones.

The main questions, which are addressed in this paper, are: (i) which is the most suitable model for the Faraday rotation measurements on JET and the possible improvement to the equilibrium calculation which can be obtained through the comparison between the measurements and the numerical solution of Stokes equations (see sections 3 and 4); (ii) how the coupling acts on Faraday and Cotton-Mouton, i.e. whether the dependence found using the type II solution is general (see section 5). Both points are relevant to the modelling of Faraday rotation and Cotton-Mouton effects to be used as constraints in equilibrium codes.

To answer the previous questions, the data from a JET polarimetric system are used: few discharges are studied which are representative of regimes where the polarimetric effects are reasonably strong (see tables 1 and 3).

The JET interfero-polarimeter is a Mach-Zehnder interferometer [1] where the analysis of the polarimetry has been added; it has a DCN laser working at a wavelength of $195 \mu \mathrm{m}$, and eight channels, four vertical channels crossing the poloidal plane at radial coordinates $R(\mathrm{~m})=1.889,2.701,3.039,3.738$; and four horizontal channels crossing the poloidal plane at different angles. In the following, the data from channels $3(R=3.039 \mathrm{~m}$, crossing the 
Table 1. Plasma parameters.

\begin{tabular}{llllllllll}
\hline Shot \# & $\begin{array}{l}n_{\mathrm{e} \min }\left({ }^{\mathrm{a}}\right) \\
\left(10^{19} \mathrm{~m}^{-3}\right)\end{array}$ & $\begin{array}{l}n_{\mathrm{e} \max }\left(^{\mathrm{a}}\right) \\
\left(10^{19} \mathrm{~m}^{-3}\right)\end{array}$ & $\begin{array}{l}\int n_{\mathrm{e}} \mathrm{d} l \mathrm{Ch} 3\left(^{\mathrm{b}}\right) \\
\left(10^{19} \mathrm{~m}^{-2}\right)\end{array}$ & $\begin{array}{l}T_{\mathrm{e} \min } \\
(\mathrm{keV})\end{array}$ & $\begin{array}{l}T_{\mathrm{e} \max } \\
(\mathrm{keV})\end{array}$ & $B_{\mathrm{T}}$ & $\begin{array}{l}I_{\mathrm{P}} \\
(\mathrm{MA})\end{array}$ & $W_{1}$ & $W_{3}$ \\
\hline 60980 & 2 & 3.9 & $4-9$ & 1.5 & 3.1 & $1.6 / 2.4$ & $2 / 1.6$ & 0.016 & 0.25 \\
67777 & 2.7 & 12 & $6-28$ & 2.5 & 3.5 & 2.7 & 2.5 & 0.11 & 1.4 \\
75238 & 3 & 10 & $18-20$ & 5 & 6 & 2.75 & 3.4 & 0.08 & 1.4 \\
\hline
\end{tabular}

${ }^{a}$ Measured values of electron density by LIDAR Thomson scattering.

${ }^{\mathrm{b}}$ Line integrated density interval as measured by the FIR interferometer, on channel 3.

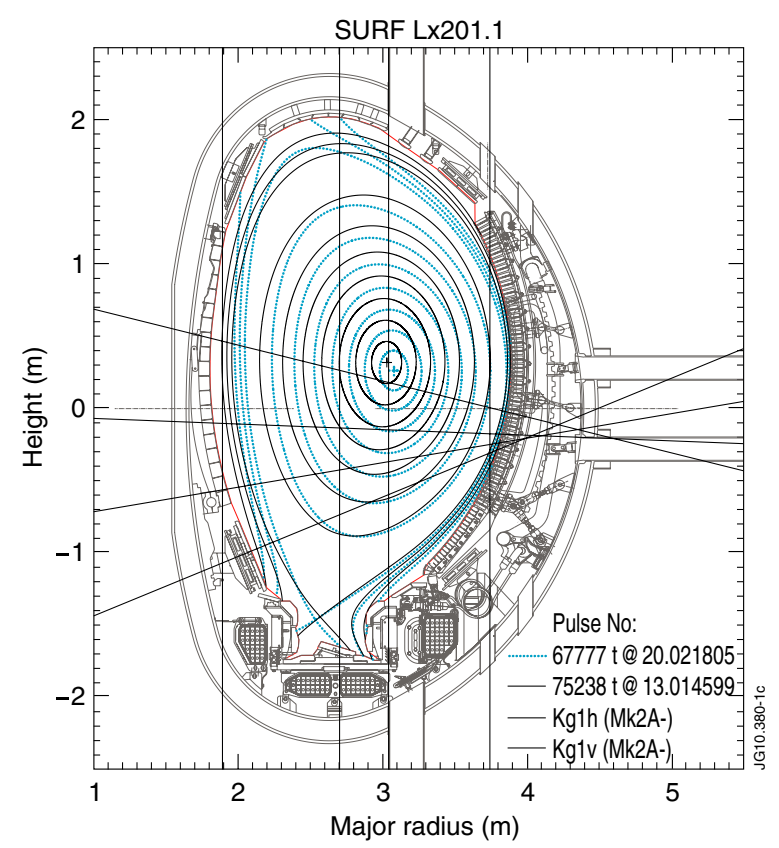

Figure 1. Equilibrium contours for shots \#67777 and 75238 and positions of the line of sights of the JET interfero-polarimeter.

plasma centre) and 4 ( $R=3.738 \mathrm{~m}$, approximately tangent to the last closed surface) will be used (see figure 1).

This paper presents a comprehensive study of the following two aspects.

(I) A detailed analysis of Faraday measurements at JET and comparison with available models (mostly approximate solutions of the Stokes equations, see section 2). It turns out that the numerical solutions of Stokes equations are in broad agreement with the Faraday rotation angle measurements on JET, inside the error bar (which is $0.02^{\circ}$ ). In general to get this agreement, a rigid radial shift of the magnetic surfaces is needed: this is demonstrated as being useful for the centre as well as edge plasma. A specific study shows that the comparison between model calculations and measurements leads to a more refined identification of the radial positions of the magnetic surfaces as predicted by the EFIT equilibrium code [3]: being the radial resolution of EFIT calculations of the order of $0.075 \mathrm{~m}$ (see section 4), this study leads to a correction of the positions of the magnetic surfaces in the range $0.02-0.05 \mathrm{~m}$. This evaluation is in broad agreement with soft-x-ray diagnostics and preliminary measurements made using an infrared camera, in particular 
the difference, with respect to EFIT evaluation, of the plasma centre position measured by soft-X and IR camera measurements of the strike points on the external divertor targets, is close to the shift of the magnetic surfaces obtained using polarimetry.

(II) A rigorous approach to the interaction between Faraday and Cotton-Mouton (studied in recent papers, see [2] for details): it is demonstrated that at high density and current, the Cotton-Mouton must be calculated including the dependence by Faraday rotation.

Since the solutions to the Stokes equations were discussed in [2], the names and classification of the solutions are retained in this paper, and only a short introduction to Stokes equations and solutions will be presented, the details are given in [2].

The paper is organized as follows: in section 2, a short summary of the measurements of the JET polarimetry system is given, together with the Stokes equations and their approximate solutions: an example of a comparison between Faraday rotation measurements and model calculations is presented; in section 3, the analysis of Faraday rotation measurements and a comparison with a rigorous solution of polarimetry propagation equations is used to determine an improvement of the position of the magnetic surfaces as calculated by EFIT (magnetic equilibrium code); in section 4, a statistical analysis on large databases of the determination of the position is presented; in section 5, a theoretical analysis of the coupling between Faraday and Cotton-Mouton effects and its application to study shots with high density and high plasma current is presented. The coupling between Faraday and Cotton-Mouton is analysed using a new set of equations (derived from and equivalent to the Stokes equations) which are useful to understand how the coupling acts; in section 6, comments are presented on the mathematical models of Faraday and Cotton-Mouton to be used as constraints in equilibrium codes; in section 7, the conclusions are presented.

Hereafter a plasma discharge is also named as 'shot'; and 'numerical solution' always refers to the numerical solution of the Stokes equations (see equation (2.2)), using as input to the equations, the density profile measured by Thomson scattering and the magnetic fields calculated by the EFIT equilibrium code [3] (see section 2); the terms 'Faraday' ('CottonMouton') will be used often, meaning 'Faraday rotation angle' ('Cotton-Mouton phase shift angle') measurements. In the paper the symbols: (i) $\varphi_{\mathrm{T}}$ and $\psi_{\mathrm{T}}$ are used for the CottonMouton phase shift (CM) and Faraday rotation angle (FR), respectively, obtained by numerical solutions of Stokes equations; (ii) $\varphi_{\mathrm{ex}}$ and $\psi_{\mathrm{ex}}$ are used for the corresponding CM and FR polarimetric measurements; (iii) $\varphi_{\text {TyII }}$ and $\psi_{\text {TyII }}$ are used for the quantities calculated using formulae (2.12); (iv) $W_{i}(i=1,2,3)$ are defined in equation (2.7)-(2.9) defining the type I approximation; (v) W3G is the Gunther Model A approximation, whose explicit expression is given in [2].

\section{Stokes equations and their approximate solutions}

The considered geometry includes the propagation of a laser beam along a vertical chord (taken as the $z$-axis) in a poloidal plane of a tokamak. The toroidal magnetic field $\left(\vec{B}_{t}\right)$ is taken along the $y$-axis, while the component of the poloidal magnetic field perpendicular to the propagation $z$-axis is taken along the $x$-axis (identical to the radial axis). The angle of the electric field vector of the input wave with $\vec{B}_{\mathrm{t}}$ is $45^{\circ}$, to maximize the Cotton-Mouton phase shift angle or the ellipticity of the wave.

The polarization of a beam can be described using the Stokes vector $\vec{s}$, whose components are expressed in terms of the ellipticity angle $(\chi)$ and Faraday angle $(\psi)$, or in terms of the ratio of the hortogonal components of the laser beam electric field (the ratio $E_{y} / E_{x}=\tan \alpha$ ) and their phase shift angle $(\varphi)$. 
The equations defining the Stokes vector $\vec{s}=\left(s_{1}, s_{2}, s_{3}\right)[2,4,5]$, in terms of $(\chi, \psi)$ and $(\alpha, \varphi)$ are

$$
\begin{aligned}
& s_{1}=\cos (2 \chi) \cos (2 \psi)=\cos (2 \alpha), \\
& s_{2}=\cos (2 \chi) \sin (2 \psi)=\sin (2 \alpha) \cos (\varphi), \\
& s_{3}=\sin (2 \chi)=\sin (2 \alpha) \sin (\varphi), \\
& s_{1}^{2}+s_{2}^{2}+s_{3}^{2}=1 .
\end{aligned}
$$

In terms of the polarization ellipse, $\psi$ (the Faraday rotation angle) is the azimuth of the polarization ellipse $(0 \leqslant \psi \leqslant \pi), \chi$ is the ellipticity angle given by $\tan \chi= \pm b / a$ $(-\pi / 4 \leqslant \chi \leqslant \pi / 4)$, where the major semiaxis of the polarization ellipse is $a$ and the minor semiaxis is $b$, and the plus sign is taken for clockwise rotation of the wave electric field, looking towards the radiation source.

The previous equations are valid when the absorption of the wave is negligible (see [8]) so that the Stokes vector is described by only three components (two of them are independent, see (2.1)).

The JET polarimeter system measures primarily (i) two components of the electric field ( $E_{x}$ and $E_{y}$, in a plane orthogonal to the propagation direction) of the laser beam emerging from the plasma as well as (ii) the phase shift $(\varphi)$ between these components. So the primary measurements are

$$
\tan \alpha=\frac{E_{y}}{E_{x}} \quad \text { and } \quad \varphi .
$$

In principle, the JET polarimetric system gives the possibility of determining directly the values of the components of the Stokes vector, using the measurements of $\alpha$ and $\varphi$ and definitions (2.1).

The Faraday rotation angle and ellipticity (defined as $\varepsilon=\tan \chi$ ) are obtained from equation (2.1):

$$
\begin{aligned}
& \tan 2 \psi=\tan 2 \alpha \cos \varphi, \\
& \tan 2 \chi=\frac{s_{3}}{\sqrt{1-s_{3}^{2}}} .
\end{aligned}
$$

The spatial evolution along the $z$-axis of the polarization of a beam is given by the Stokes equation:

$$
\frac{\mathrm{d} \vec{s}}{\mathrm{~d} Z}=\vec{\Omega} \times \vec{s}
$$

where

$$
\vec{\Omega}=k a\left(\Omega_{1}, \Omega_{2}, \Omega_{3}\right) \quad \text { and } \quad \Omega_{1}=C_{1} n\left(B_{\mathrm{t}}^{2}-B_{x}^{2}\right), \quad \Omega_{2}=2 C_{1} n B_{x} B_{\mathrm{t}}, \quad \Omega_{3}-C_{3} n B_{z}
$$

where $B_{\mathrm{t}}$ is the toroidal magnetic field (Tesla), $B_{z}$ is the component of the poloidal magnetic field along the propagation axis, $B_{x}$ is the component of poloidal magnetic field orthogonal to the propagation axis (the ratio $B_{x} / B_{\mathrm{t}} \leqslant 10^{-1}$, so neglecting $B_{x}$ implies an error $\leqslant 1 \%$ in the evaluation of $\left.\Omega_{1}\right), n$ is the electron density $\left(\mathrm{m}^{-3}\right), C_{1}=1.8 \times 10^{-22}$ and $C_{3}=2 \times 10^{-20}$, calculated for the laser wavelength of $\lambda=195 \mu \mathrm{m}$, and $Z=z / k a$ is the normalized coordinate along a vertical chord, where $k$ is the elongation and $a$ is the minor radius. The relations between the Faraday rotation $\psi$ and the Cotton-Mouton phase shift $\varphi$ angles and the corresponding components of Stokes vector follow from (2.1):

$$
\begin{aligned}
& \frac{s_{2}}{s_{1}}=\tan 2 \psi \\
& \frac{s_{3}}{s_{2}}=\tan \varphi .
\end{aligned}
$$


Table 2. Error bars of measurements used in the calculations.

\begin{tabular}{llll}
\hline Diagnostic system & Measured quantity & Symbol & Error bar \\
\hline Polarimeter & Faraday rotation & $\psi$ & $0.2^{\circ}$ \\
Polarimeter & Cotton-Mouton phase shift & $\varphi$ & $2^{\circ}$ \\
LIDAR Thomson scattering & Line-integral plasma density & $\int n e \mathrm{~d} L$ & $10 \%$ \\
LIDAR TS & Plasma density profile & $n_{\mathrm{e}}$ & $5 \%$ \\
LIDAR TS & Plasma temperature profile & $T_{\mathrm{e}}$ & $10 \%$ \\
\hline
\end{tabular}

Equation (2.2) is solved, with the initial condition (i.e. the Stokes vector of the input wave):

$$
\vec{s}_{\text {input }}=(0,1,0)
$$

corresponding to a $45^{\circ}$ angle between the electric field vector of the input wave and $\vec{B}_{\mathrm{t}}$. This arrangement is optimized for Cotton-Mouton measurements.

In this work data related to the channels 3 and 4 are presented: the data exhibit a reasonably good signal to noise ratio and the geometry to be analysed is relatively simple. Figure 1 shows for shots \#67777 and \#75238 the equilibrium contours and the positions of the line of sights of the vertical and horizontal channels of the polarimeter, channel 3 is the vertical line at $R=3.039 \mathrm{~m}$, while (vertical) channel 4 has the radial coordinate at $R=3.738 \mathrm{~m}$.

The values of the vector $\vec{\Omega}=k a\left(\Omega_{1}, \Omega_{2}, \Omega_{3}\right)$ are obtained [2] using the values of $\vec{B}$ as calculated by the EFIT equilibrium reconstruction and the LIDAR Thomson scattering measurements of plasma density $(n)$ projected along the line of sight of the vertical channels on the basis of the reconstructed equilibrium.

The values of $\vec{B}$ in the following sections are determined by EFIT using mainly external (to the plasma) magnetic measurements. The question is about the accuracy of the evaluation of the magnetic field given by EFIT, and its effect on the accuracy of the calculations of Faraday rotation by solving the Stokes equations. This question is linked to the accuracy of the EFIT determination of the current profile and the related safety factor spatial profile. The accuracy of the safety factor evaluations made by EFIT could be estimated $\Delta q(0) / q(0) \leqslant 20-30 \%[12,13]$, where $q(0)$ is the safety factor at the plasma centre. The effect of the accuracy of the current profile on the interpretation of polarimetric measurements can be evaluated noting that channel 3 has a line of sight passing through the magnetic centre where the poloidal magnetic field $\left(B_{\mathrm{p}}\right)$ is inverting the sign (being $B_{\mathrm{p}}=0$ at the magnetic centre) so the determination of the Faraday is mostly influenced by the values of $B_{\mathrm{p}}$ at mid-radius where the accuracy is relatively improved and the error bar could be estimated in $\Delta B_{\mathrm{p}} / B_{\mathrm{p}}$ (mid-radius) $\leqslant 10 \%$, leading to an estimation of the accuracy of $\Delta \Omega_{i}(i=1,2,3) / \Omega_{i} \leqslant 15 \%$ (if the accuracy on the measurements of the plasma density profile, see table 2 , is included). To link this evaluation to the accuracy on the calculation of Stokes vector we note that the Stokes equations (2.2) can be written as

$$
\frac{\mathrm{d} \vec{s}}{\mathrm{~d} Z}=M \cdot \vec{s}
$$

where $M$ is a matrix containing $\Omega_{i}$. The formal solution can be expressed as $s_{i}=$ $\exp \left(\int M_{i j} \mathrm{~d} Z\right) \cdot s 0_{j}$ which implies that the error bar on the values of the Stokes vector can be evaluated as $\Delta s / s \leqslant \max (\Delta M / M) \leqslant \Delta \Omega_{i}(i=1,2,3) / \Omega_{i} \sim 15 \%$. The accuracy on the equilibrium calculation has the effect on the calculation of the Faraday rotation angle leading to the rough evaluation of $\Delta \psi / \psi \sim \Delta s / s \leqslant 15 \%$ (using equation (2.4), and the approximation of $\tan 2 \psi \sim 2 \psi$ ). 
The type I solution [2,5] is obtained as the first term of a series expansion in $W_{i}=$ $\int \Omega_{i} \mathrm{~d} z, W_{i}^{2} \ll 1(i=1,2,3)$, to the solution of the system of ordinary differential equations (2.2), together with the initial condition (2.6). In this approximation the relations between the Stokes vector, the Faraday rotation $\psi$ and Cotton-Mouton phase shift angle $\varphi$ are given by

$$
\begin{aligned}
& s_{1}=-W_{3}=C_{3} \int n_{\mathrm{e}} B_{z} \mathrm{~d} z=1 / \tan 2 \psi, \\
& s_{2}=1-\left(W_{1}^{2}+W_{3}^{2}\right) / 2 \approx 1, \\
& s_{3}=W_{1}=C_{1} \int B_{\mathrm{t}}^{2} n_{\mathrm{e}} \mathrm{d} z=\tan \varphi .
\end{aligned}
$$

Relations (2.7) and (2.9) are the key equations used for evaluating the polarimetric measurements linking the Faraday rotation to the component of the poloidal magnetic field along the direction of propagation of the laser beam (and then to the plasma current profile), and the Cotton-Mouton phase shift angle to the line integral of the electron density. The expressions in (2.7) and (2.9) are valid only for $W_{i}^{2} \ll 1$ : for large Faraday and Cotton-Mouton angles (see also in the following the discussion on type II approximation) other methods must be used to find solutions to the Stokes equations. The term 'linear approximation' will be used in this paper with reference to formulae (2.7)-(2.9).

The physical meaning of the type I approximation can be appreciated, if we consider the situation where the transverse components of the magnetic field are not present (i.e. $B_{\mathrm{t}}=B_{x}=0$ in (2.3), and $\left.\Omega_{1}=\Omega_{2}=0\right)$, and there is a magnetic field $B_{z}$ in the direction of beam propagation. This is the case of a 'pure' Faraday rotation. Solving the Stokes equations (2.2) with the initial conditions (2.6) leads to the solutions:

$$
\begin{aligned}
& s_{1}=\cos 2 \psi=\cos \left(2 \psi_{0}+W_{3}\right), \\
& s_{2}=\sin 2 \psi=\sin \left(2 \psi_{0}+W_{3}\right), \\
& s_{3}=\sin 2 \chi_{0}=0,
\end{aligned}
$$

the Faraday rotation is then obtained from the previous equations:

$$
2 \psi=2 \psi_{0}+W_{3} .
$$

It can be verified that the previous formula leads to (2.7) (type I) at the same level of the approximation (i.e. $W_{3} \ll 1$, in practice $W_{3} \ll \pi / 6=0.5$ ). The 'pure' Faraday rotation is represented by $W_{3} / 2$.

The 'pure Cotton-Mouton' can be derived from the solutions of Stokes equations considering the case of $B_{z}=B_{x}=0$, i.e. $\Omega_{3}=0=\Omega_{2}$ :

$$
\begin{aligned}
& s_{1}=s_{10}=0, \\
& s_{2}=\sin 2 \alpha_{0} \cos \varphi=\sin 2 \alpha_{0} \cos \left(\varphi_{0}+W_{1}\right)=\cos \left(\varphi_{0}+W_{1}\right), \\
& s_{3}=\sin 2 \alpha_{0} \sin \varphi=\sin 2 \alpha_{0} \sin \left(\varphi_{0}+W_{1}\right)=\sin \left(\varphi_{0}+W_{1}\right) .
\end{aligned}
$$

The 'pure Cotton-Mouton' is then represented by $W_{1}$; in fact from the previous equations we obtain

$$
\varphi=\varphi_{0}+W_{1}
$$

Under the ideal conditions $\varphi_{0}=0$, so $\varphi=W_{1}$.

The physical meaning of $W_{1}$ and $W_{3}$ is related to 'pure' Cotton-Mouton and Faraday rotation, respectively, in a context where the two effects can be considered separately, independent and small. 
More general approximate solutions [2] to equation (2.2) can be found, observing that the following inequalities between the components of the vector $\vec{\Omega}$ hold for tokamak plasmas:

$$
\left|\Omega_{3}\right| \geqslant \Omega_{1}>\left|\Omega_{2}\right| \text {. }
$$

As a consequence of condition (2.10), the terms with component $\Omega_{2}$ can be neglected in the Stokes equations (2.2) and terms in $\Omega_{1} s_{3}$ neglected with respect to $\Omega_{3} s_{1}$, in these approximations the Stokes equations can be integrated analytically, and the expressions (type II solutions) for the Faraday angle and Cotton-Mouton phase shift can be obtained [2]:

$$
\begin{aligned}
& \frac{s_{2}}{s_{1}}(z)=\tan 2 \psi=-\frac{1}{\tan \left(W_{3}\right)}=\tan \left(W_{3}+2^{*} \pi / 4\right), \\
& \frac{s_{3}}{s_{2}}(z)=\tan \varphi=\frac{\int_{-z}^{+z} \mathrm{~d} y \Omega_{1}(y) \cos \left(W_{3}(y)\right)}{\cos \left(W_{3}(z)\right)} .
\end{aligned}
$$

A clear trend present in formulae (2.12) is that the Cotton-Mouton phase shift increases with $W_{3}$ (for values corresponding to JET data) (see section 3 and figure 6). In practice for Faraday rotation angles $\psi-\psi_{0} \leqslant 12^{\circ}$ (the initial angle is $\psi_{0}=\pi / 4$, see (2.6)), $1 \geqslant \cos \left(W_{3}\right) \geqslant 0.9$ and $\tan \varphi \approx W_{1}$, within an approximation of $10 \%$, whereas for Faraday angles $\psi-\psi_{0} \geqslant 12^{\circ}$ the Cotton-Mouton increases due to the enhancement linked with Faraday rotation $\left(W_{3} \geqslant 2 \pi / 15=0.4\right)$.

In the following discussion concerning the comparison between measurements and model calculations, the Guenther Model A [4] will be cited as well: a discussion of the details of this model is given in [2,5]. This model is the analytic solution of Stokes equations in the limit of $\Omega_{3} / \Omega_{1} \sim B_{z} / B_{x}^{2}=K=$ constant.

Examples of the calculations of the Faraday effect can be produced, starting from data of shots representative of JET typical operational space. Table 1 gives a choice of three shots with parameters from low density/low current, to high density/high current. Table 2 summarizes the values of the experimental errors in the measurements used in the Stokes equations.

We start with a low density shot (\#60980): the case of 'pure' Faraday effect. In particular, type $\mathrm{I}$ is expected to fit well the data and this is due to the low value of the Faraday angle and corresponding $\mathrm{W}_{3}$. Figure $2(a)$ shows the numerical solution of Stokes equations $\left(1 / \tan 2 \psi_{\mathrm{T}}\right)$, together with the Faraday measurements $\left(1 / \tan 2 \psi_{\mathrm{ex}}\right)$ for shot \#60980: the numerical solution agrees with measurements within the error bar, which for Faraday rotation angle is $\Delta \psi=0.004 \mathrm{rad}\left(0.22^{\circ}\right)$. The data shown on the plot are taken every $840 \mathrm{~ms}$. A $\chi^{2} / n=0.9$ ( $n$ is the number of data) is calculated: the model fits well the data. Figure $2(b)$ shows the plasma parameters of shot \#60980: time evolution of the line integrated electron density on channel 3 measured by the interferometer, maximum temperature as measured by LIDAR Thomson scattering, toroidal magnetic field and plasma current. The strong dynamics of the polarimeter at time $=15 \mathrm{~s}$ is reflected by the variation of the interferometer.

Figure 3 shows the comparison between Faraday measurements and the approximate solutions (type I (2.7-9), type II (2.12) and Guenther Model A (W3G)) for the low density shot \#60980. All the models are in agreement with the measurements, with different levels of accuracy: the values of the $\chi^{2} / n=1.33$ (type II), 1.46 (type I) and 0.0025 (Guenther Model A). The Guenther Model A fits well the data.

Effects on polarization state due to dichroism (differential absorption of the characteristic waves propagating inside plasma) were analysed (see [8]): for JET plasma parameters the absorption of the injected wave is negligible and calculations show that the order of magnitude of the matrix elements corresponding to dichroism are a factor of $10^{-9}$ with respect to values of $\Omega_{1,3}$, appearing in the Stokes equations. 

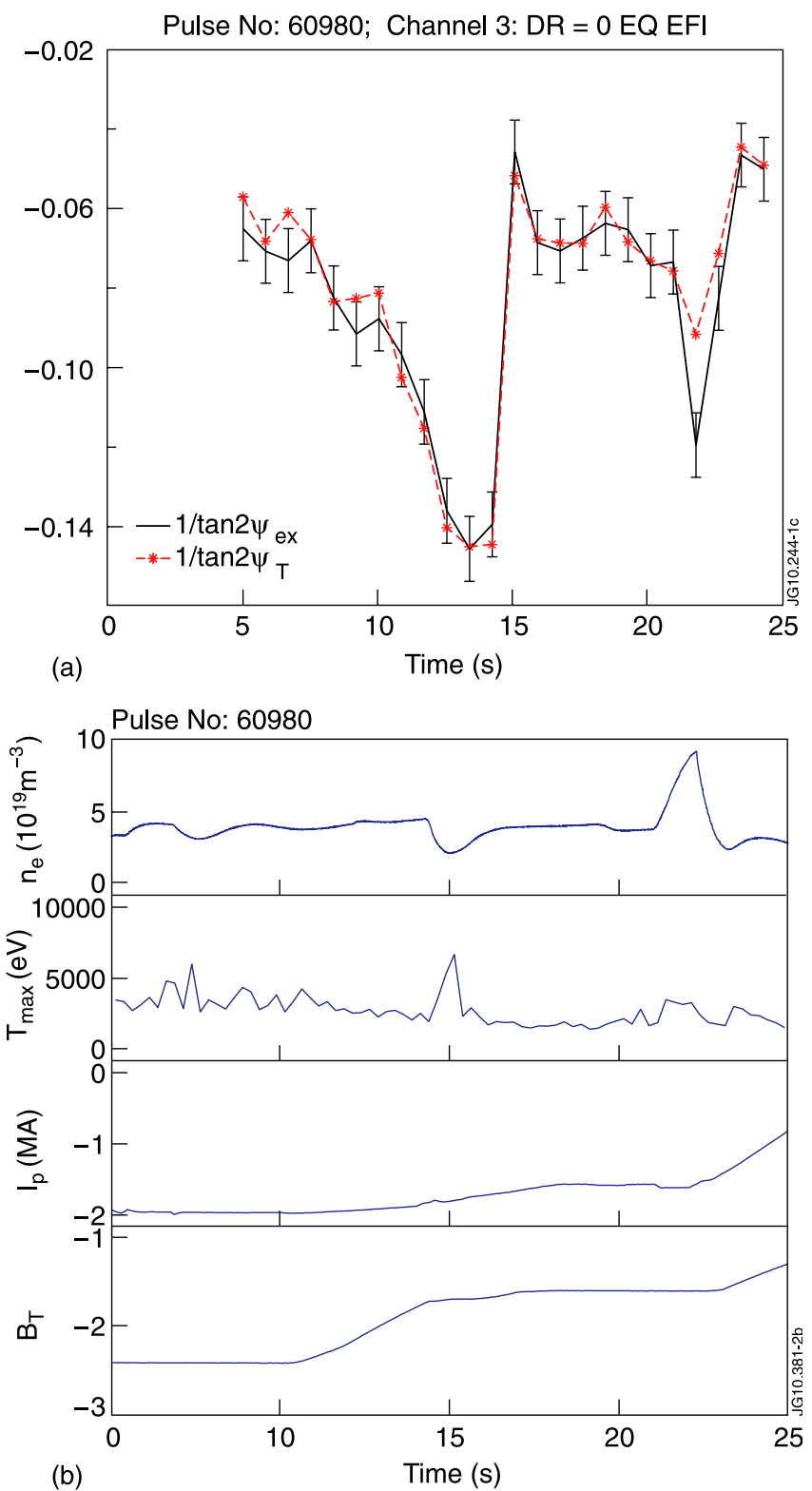

Figure 2. (a) Faraday rotation measurement (continuous line, shot \#60980, channel 3) is plotted together with the calculated values ('*' symbol) using the numerical solution of Stokes equations. Plasma parameters are given in table 1. Agreement within the error bars. (b) Plasma parameters of shot \#60980, from top: measurements of line integrated density by the interferometer channel 3 shot \#60980; Electron temperature measured by LIDAR Thomson scattering; plasma current (MA); toroidal magnetic field (T).

\section{Faraday rotation measurements and EFIT equilibrium evaluation}

The calculation of the Faraday rotation angle using the Stokes model is very sensitive to the components of the magnetic field used in the numerical solution of equation (2.1): it is less 


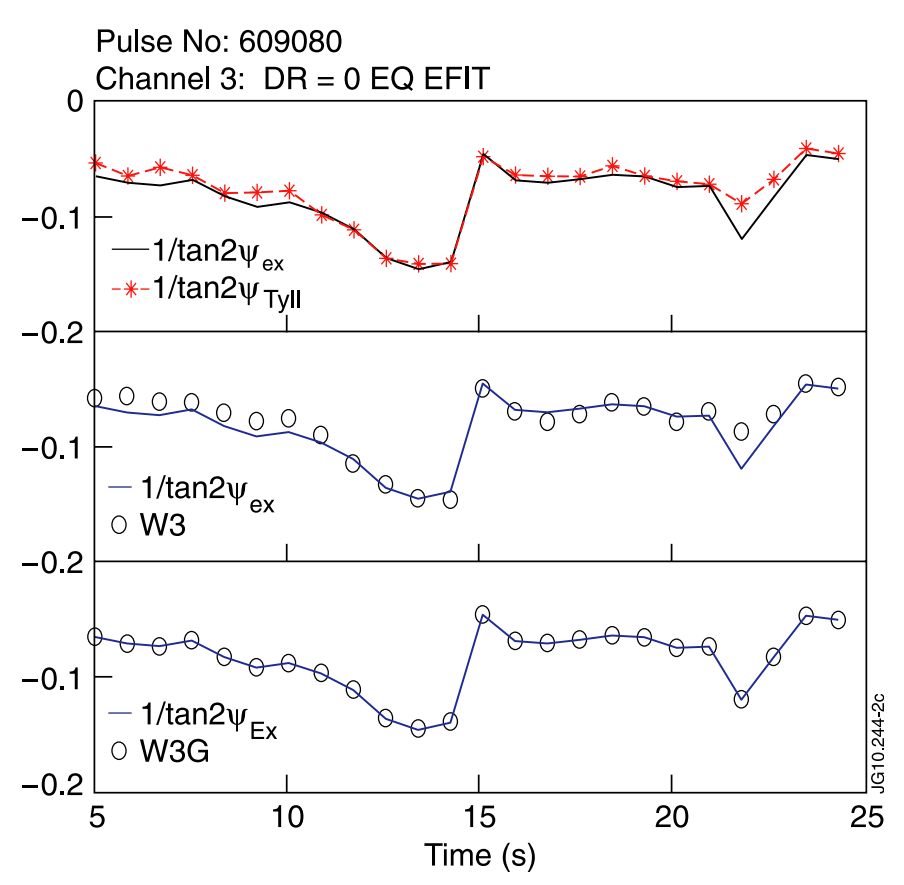

Figure 3. Faraday rotation. A complete comparison (and agreement) between the models and measurements is presented for shot \#60980, channel 3. From the top the comparison of measurements with the type II approximation, the 'linear' $\mathrm{W}_{3}$ approximation, and the Guenther Model A.

sensitive to the density profile. In this sense the comparison between calculations and data can be used to check the accuracy of the equilibrium calculations. The argument can be motivated in detail as follows. The vector $\vec{\Omega}(z, x)$ appearing in the Stokes equations depends upon the values of the magnetic field components $(\vec{B}(z, x))$ and plasma density spatial profiles $\left(n_{\mathrm{e}}(z, x)\right)$ ( $x$ is the radial direction). However, there is a different sensitivity upon $\vec{B}(z, x)$ and $n_{\mathrm{e}}(z, x)$. The plasma density changes slowly in the radial direction, so $n_{\mathrm{e}}(z, x) \approx n_{\mathrm{e}}(z, x+\mathrm{d} x)$ if $\mathrm{d} x / a \ll 1$, while the magnetic field changes substantially $\vec{B}(z, x) \neq \vec{B}(z, x+\mathrm{d} x)$. For example in shot $\# 76846$ for a $\mathrm{d} x=0.07 \mathrm{~m}$ the plasma density profile $n_{\mathrm{e}}(z, x)$ does not change but the $B_{z}(z, x)$ changes by more than $50 \%$ at the plasma centre.

Figure 4 shows the calculations of the Faraday rotation for the high density shot \#67777 corresponding to channel 3 . The values of the spatial profile of $B_{z}\left(z, R_{\text {channel }}\right)\left(R_{\text {channel }}\right.$ is the radial coordinate of the vertical chord and $z$ the coordinate along the chord) given by the equilibrium calculation are critical for the determination of the Faraday rotation: we find that the equilibrium in agreement with the measurements of polarimetry corresponds to a shift $\mathrm{DR}=0.035 \mathrm{~m}$ of the magnetic surfaces in the direction of high-field side. Under these conditions we find that all the models (also the 'linear' model $\mathrm{W}_{3}$ ) are in agreement with the measurements. The same procedure must be applied to the calculations related to channel 4 which is the outermost vertical channel with coordinate $R=3.74 \mathrm{~m}$, leading to a shift DR $=0.04 \mathrm{~m}$ (for shot \#67777). This is not expected because the equilibrium is supposed to be accurate at plasma edge, where the magnetic sensors are placed. Data related to channel 4 are shown in figure 5 from the top: the first plot shows a comparison between the numerical calculations and measurements, the second plot shows the measurements compared with type II model and the third shows the comparison between the measurements and type I 


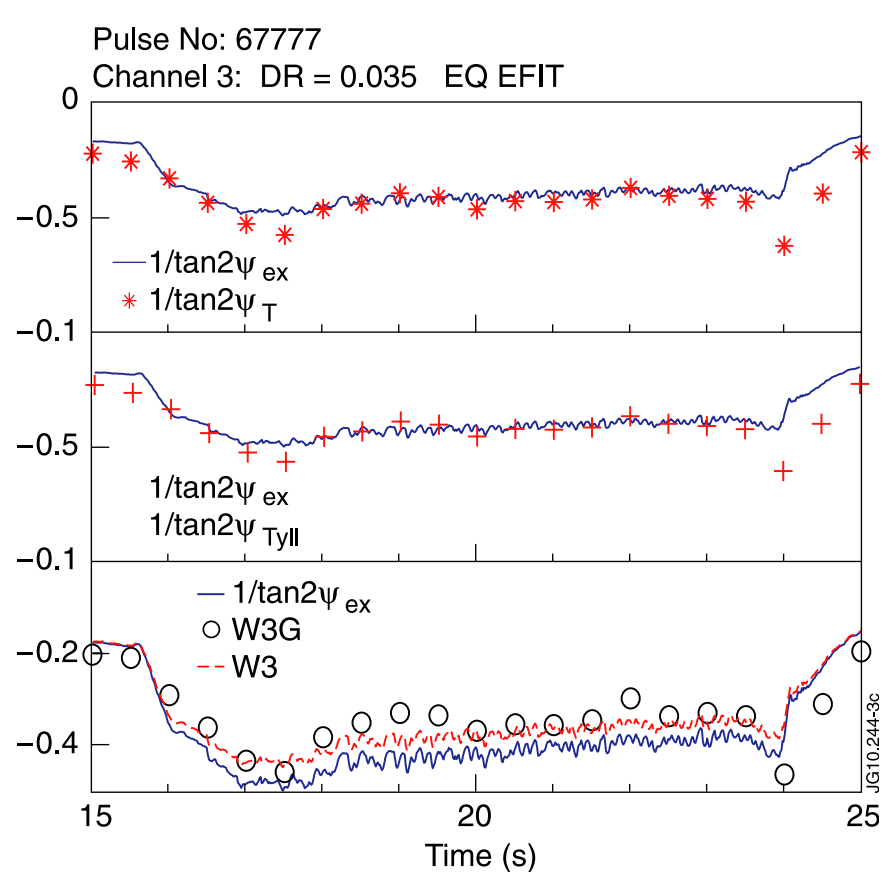

Figure 4. Faraday rotation. Similar to figure 3, but for the high density shot \#67777, channel 3: the third figure from the top reports the calculation of $\mathrm{W}_{3}$ (the linear approximation) and of $\mathrm{W} 3 \mathrm{G}$ (Guenther Model A): here the agreement between data and models is obtained by a rigid shift of the magnetic surfaces DR $=0.035 \mathrm{~m}$. The data are in agreement with all the models, in particular with the 'linear' $\mathrm{W}_{3}$ model.

and Guenther Model A. In practice, the numerical model and type II are in agreement with data, while type I and the Guenther Model A underestimate the Faraday measurements. The Faraday measurements give quite a large rotation (as it can be expected) on channel 4 of the order of $23^{\circ}$, and a value of $W_{3} \approx 1$ : we find in fact that type I (which is a measurement of 'pure Faraday' rotation) is not a good approximation. This experimental finding confirms the conclusions drawn from the type II model in section 2.

The equilibrium used in the previous calculations is evaluated including only magnetic measurements, the question arises as to whether an equilibrium evaluated using constraints from polarimetry could improve the prediction of Faraday rotation as calculated by the Stokes equations, without needing a shift. To answer this question an equilibrium was generated by EFIT where the minimization was obtained including the constraint of Faraday rotation, but a shift of $0.035 \mathrm{~m}$ was still needed to reconcile the measurement with the calculations. One possible reason is that the mathematical form of the polarimetry constraint used in the EFIT code is a simplified type I approximation (see equation (2.10)) corresponding to the 'pure Faraday effect': for large Faraday rotations this approximation is marginal.

The question is now whether the shift of the magnetic surfaces would decrease using an equilibrium (considered more precise) generated by EFIT constrained with internal measurements.

To study further this problem comparisons between data and calculations were carried out for shot \#76846( $\left.B_{\mathrm{T}} / I_{\mathrm{p}}=1.7 \mathrm{~T} / 1.4 \mathrm{MA}, n_{\mathrm{e}}=5.5 \times 10^{19} \mathrm{~m}^{-3}, T_{\mathrm{e}}=4.5 \mathrm{keV}\right)$, with two types of equilibria: (i) the EFIT equilibrium obtained using only magnetic measurements (IEFIT); (ii) the EFIT equilibrium obtained using constraints which include motional Stark 


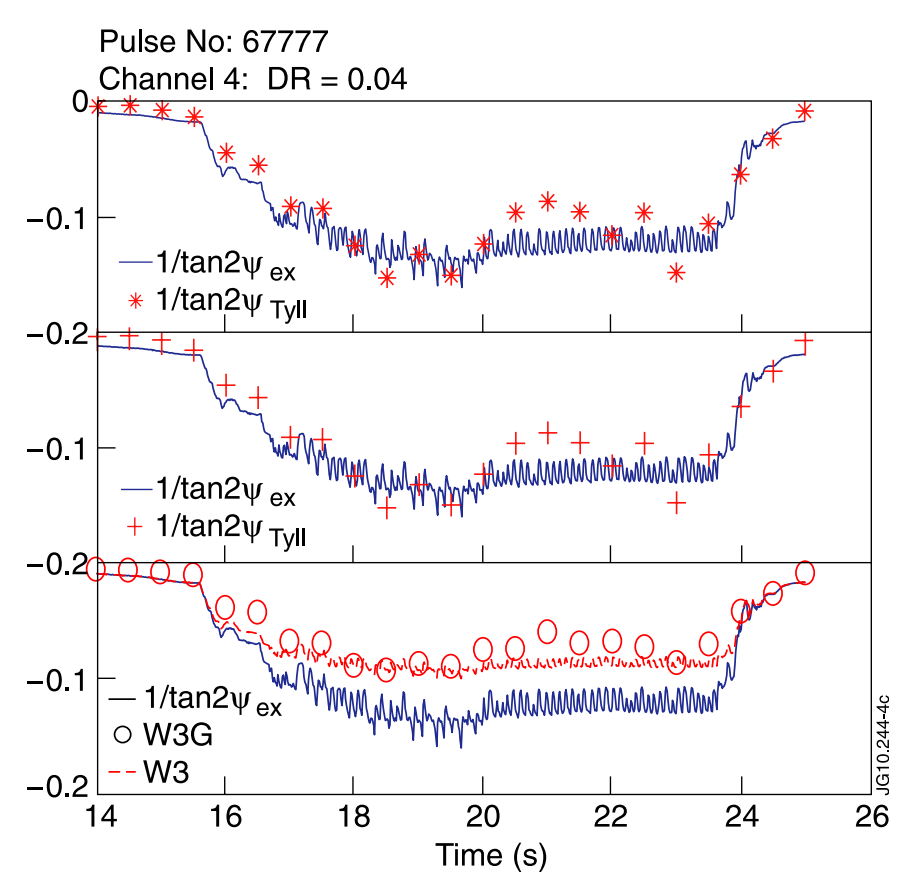

Figure 5. Faraday rotation. Comparison between models and data for shot \#67777, channel 4: from the top the comparison of measurements with numerical solution, the type II approximation, the 'linear' $W_{3}$ approximation and the Guenther Model A. Agreement between data and the Stokes numerical solutions and the type II approximation is found operating a rigid shift of $0.04 \mathrm{~m}$ of the magnetic surfaces. The approximate values of $\mathrm{W}_{3}$ (and Guenther Model A) slightly underestimate the measurement.

effect (MSE) measurements and pressure measurements (performed by the high resolution Thomson scattering (HRTS) system) (EFTM). Figure 6(a) shows the comparison between calculations and data using IEFIT, and figure $6(b)$ shows the results of comparison when the shift DR $=0.045 \mathrm{~m}$ is introduced, using the IEFIT equilibrium. Figure $6(c)$ shows the comparison (between Faraday data and calculations) using the constrained equilibrium EFTM: it can be noted that using EFTM an improvement is obtained, but this is not enough to obtain an agreement between measurements and calculations. The agreement is found only introducing a rigid shift of the magnetic surfaces of DR $=0.02 \mathrm{~m}$ when EFTM is used (see figure $6(d)$ ). Figure 6 corresponds to measurements of channel 3.

Thus using a more refined equilibrium such as EFTM, which includes internal measurements (MSE and pressure profiles) as constraints, leads to a reduction in the shift of the magnetic surfaces $(\mathrm{DR}=0.02 \mathrm{~m}$ instead of $\mathrm{DR}=0.04 \mathrm{~m}$ ) needed to reconcile polarimetry model and measurements.

In more detail, the previous figures show that: (i) the comparison between the calculations and measurements of Faraday rotation can give important information about the accuracy of equilibrium calculations; (ii) the comparison can be used to improve the calculations of the position of the equilibrium surfaces: in practice, the calculation of the Faraday rotation angle using the Stokes model is useful to evaluate how to shift the flux surfaces to improve the evaluation of the equilibrium.

An evaluation of refraction effects leads to maximum values of $0.004 \mathrm{~m}$ of a beam deflection for a ray path of $4 \mathrm{~m}$ inside plasma at plasma density $n=0.5 \times 10^{20} \mathrm{~m}^{-3}$, this means that the shift found cannot be caused by refraction effects. 

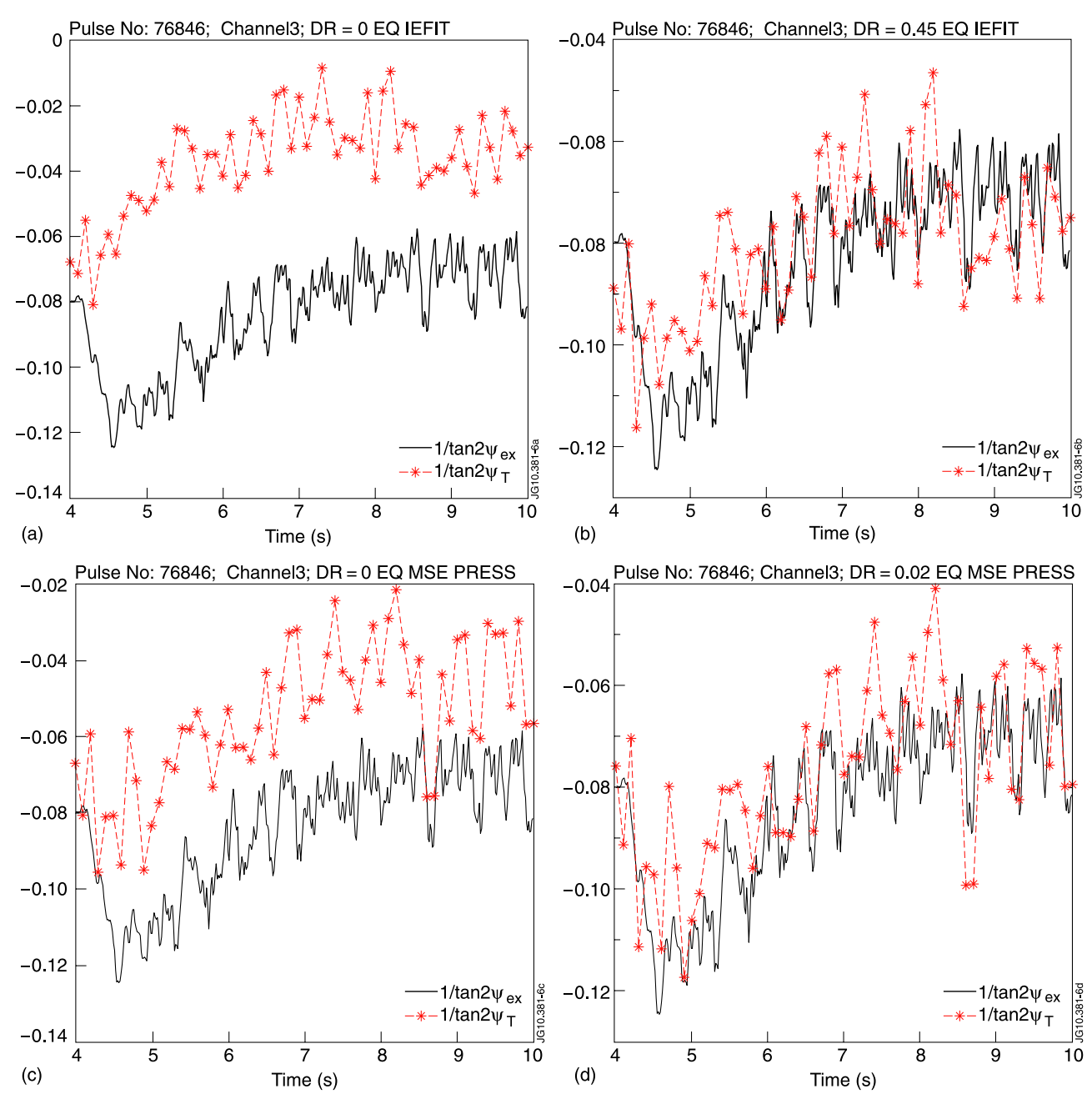

Figure 6. ( $a$ ) Faraday rotation. Comparison between measurements (continuous line) and results of the numerical solution of Stokes equations (dashed line with stars) for shot \#76846, where the shift of magnetic surfaces DR $=0$. The EFIT equilibrium (which includes magnetic measurements) is used without constraints (EQ IEFIT). (b) Faraday rotation. Comparison between measurements (continuous line) and results of the numerical solution of Stokes equations (dashed line with stars) for shot \#76846, where the shift of magnetic surfaces DR $=0.045 \mathrm{~m}$. The EFIT equilibrium is used without constraints (EQ IEFIT). (c) Faraday rotation. Comparison between measurements (continuous line) and results of the numerical solution of Stokes equations (dashed line with stars) for shot \#76846, where the shift of magnetic surfaces DR $=0$. The EFIT equilibrium is used with constraints including motional Stark effect (MSE) and pressure profile measurements (EFTM). (d) Faraday rotation. Comparison between measurements (continuous line) and results of the numerical solution of Stokes equations (dashed line with stars) for shot \#76846, where the shift of magnetic surfaces DR $=0.02 \mathrm{~m}$. The EFIT equilibrium is used with constraints including MSE and Thomson scattering pressure profile measurements (EFTM).

To verify the consistency of the correction to the equilibrium obtained by analysis of polarimetry and measurements of other diagnostics, we have analysed the measurements of the strike points using IR cameras and the position of the plasma centre measured by soft-X.

The shifts of magnetic surfaces evaluated are consistent with measurements of the IR camera of the strike points on the external divertor targets: preliminary evaluations lead to 
Table 3. Dataset used to study the dependence of the shift upon plasma parameters: $n_{\max }$ maximum electron density in units of $10^{20} \mathrm{~m}^{-3} ; I_{\mathrm{P}}$ - plasma current in MA; $B_{\mathrm{T}}$ - toroidal magnetic field in Tesla.

\begin{tabular}{llll}
\hline & $n_{\max }$ & $I_{\mathrm{P}}$ & $B_{\mathrm{T}}$ \\
\hline $70053^{\mathrm{a}}$ & 1.35 & 3.56 & 3.14 \\
$70222^{\mathrm{a}}$ & 1.22 & 3 & 2.99 \\
70238 & 0.97 & 2.55 & 2.63 \\
$70646^{\mathrm{a}}$ & 0.93 & 2.28 & 2.18 \\
70691 & 0.9 & 1.85 & 2.26 \\
$68515^{\mathrm{a}}$ & 0.9 & 1.73 & 1.83 \\
$68741^{\mathrm{a}}$ & 0.87 & 1.73 & 1.83 \\
$70004^{\mathrm{a}}$ & 0.63 & 2.52 & 2.55 \\
70206 & 0.41 & 1.22 & 1.9 \\
70275 & 0.5 & 1.95 & 3.09 \\
70312 & 0.43 & 1.62 & 2.96 \\
70084 & 0.38 & 1.8 & 3.36 \\
70558 & 0.28 & 2 & 2.31 \\
70336 & 0.6 & 1.9 & 3 \\
$70548^{\mathrm{a}}$ & 0.6 & 2 & 2.7 \\
\hline
\end{tabular}

a shots included in the analysis reported in figure 7.

a difference of $0.02-0.05 \mathrm{~m}$ between the evaluation of position carried out by EFIT and the measurement made using the IR camera [9].

A comparison of plasma centre position measurements using soft-x-ray peak emission and EFIT evaluation for shot \#67777 was carried out: the average difference, in the time window $[17,21] \mathrm{s}$, between EFIT and soft-X is DR $=0.048 \mathrm{~m}=\mathrm{R}(\mathrm{EFIT})-R$ (soft-X) [10]. This difference is consistent with the shift evaluated for shot \#67777, using the comparison between the numerical code and the Faraday rotation angle measurements.

The same comparison was carried out for seven shots chosen in table 3 (varying current and density) and in figure 7 the difference between the plasma centre measured by soft-X and calculated by EFIT ( $\left.R_{\mathrm{sxr}}-R_{\mathrm{mhd}}\right)$ is plotted together the shifts calculated using polarimetry measurements, for seven shots whose plasma parameters are reported in table 3 . The result shows that there is a consistency (within the error of the measurements) between the calculations of the shifts and the measurements of the difference $R$ (soft-X)- $R$ (mhd). In figure 7 the shift (and its error bar) was calculated minimizing the $\chi^{2}$ between measurements and calculation.

The accuracy of the EFIT equilibrium reconstruction has been analysed in [12] in the context of the determination of the safety factor spatial profile using the equilibrium code constrained by MSE and pressure profile measurements, with the addition of Faraday rotation angle constraint. A type I solution simplified constraint, i.e. $\psi=\int n \vec{B} \cdot \mathrm{d} \vec{l}$ is used in EFIT (the line integral is along the chord, $\psi$ is the measured Faraday angle, $n$ is the density profile and $B$ is the magnetic field vector) properly for a shot where the Faraday rotation is very low, $\psi=3^{\circ}$. It is worth remembering that the simplified constraint is used in EFIT in general, and is not limited at low values of Faraday rotation angles.

\section{Statistical analysis of the 'shift' of the magnetic surfaces}

To determine the dependence of the calculated shift upon plasma parameters, a statistical analysis was carried out on a large database of shots including measurements of years 20032007, confirming the procedure outlined for shot \#67777. In particular, a (reduced) database was built (see table 3) to study the dependence upon the main plasma parameters (plasma density, current and toroidal magnetic field), of the shift of the magnetic surfaces needed to 


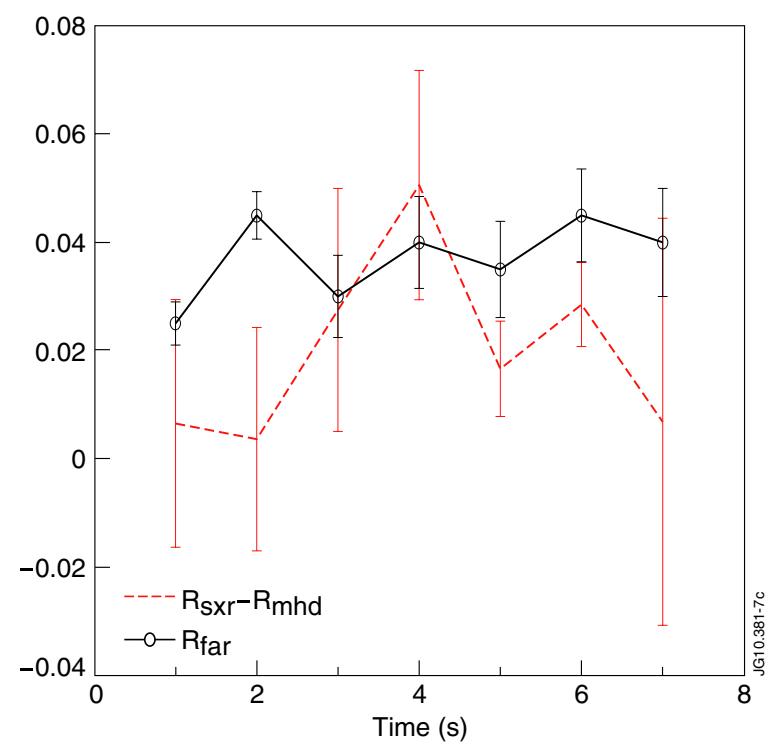

Figure 7. The difference between the plasma centre measured by soft- $\mathrm{X}$ and calculated by EFIT $\left(R_{\mathrm{sxr}}-R_{\mathrm{mhd}}\right)$ plotted together the shifts calculated using polarimetry measurements, for seven shots whose plasma parameters are reported in table 3 .

reconcile the Faraday measurements with the calculations. The study was limited to data of channel 3. The equilibrium used was EFIT with magnetic measurements only.

For each shot (see table 3), the shift was determined by the minimization of the $\chi^{2}$, developed using the Faraday measurements and the corresponding values obtained by solving the Stokes equations.

The result of the analysis is shown in figures $8(a)-(c)$ : figure $8(a)$ is a plot of the shift versus the maximum line-averaged electron density measured by LIDAR Thomson scattering; figure $8(b)$ is a plot of the shift versus the plasma current; and figure $8(c)$ shows the shift versus the ratio $B_{T} / I_{P}$ (toroidal magnetic field/plasma current).

An extensive statistical analysis (based on validated shots belonging to campaigns of years 2007-2009) on the shift was carried out for the measurements of channel 4 (the outermost vertical channel with coordinate $R=3.74 \mathrm{~m}$ ). In the calculations of the Stokes equations the density profile used were measured by the HRTS, which has a spatial resolution at edge of the order of $0.015 \mathrm{~m}$. The average shift calculated was of the order of DR $=0.02 \mathrm{~m}$.

The calculated shift, ranging in the interval DR $=0.01-0.05 \mathrm{~m}$, does not exhibit any strong dependence upon plasma parameters. We conclude from these findings that the shift needed is due to the accuracy of the EFIT equilibrium calculations, in terms of space resolution: this is limited by the dimension of the elements of the grid used by EFIT [6]: the element has a dimension $\mathrm{d} Z \times \mathrm{d} R=0.126 \times 0.075 \mathrm{~m}^{2},(\mathrm{~d} Z(\mathrm{~d} R)$ is the dimension in the vertical(radial) direction). The shift then found in this study is consistent with the radial space resolution of the EFIT calculations.

From these data it is important to realize that the comparison between calculation and measurement of Faraday rotation can lead to an improvement of the evaluation of the position of the magnetic surfaces.

To confirm that the EFIT calculations can be affected by a certain systematic uncertainty, a comparison of the average radial location of the lower outer strike point as predicted by EFIT and XLOC was done, using a large database of 460 shots chosen in years 2003-2007. 

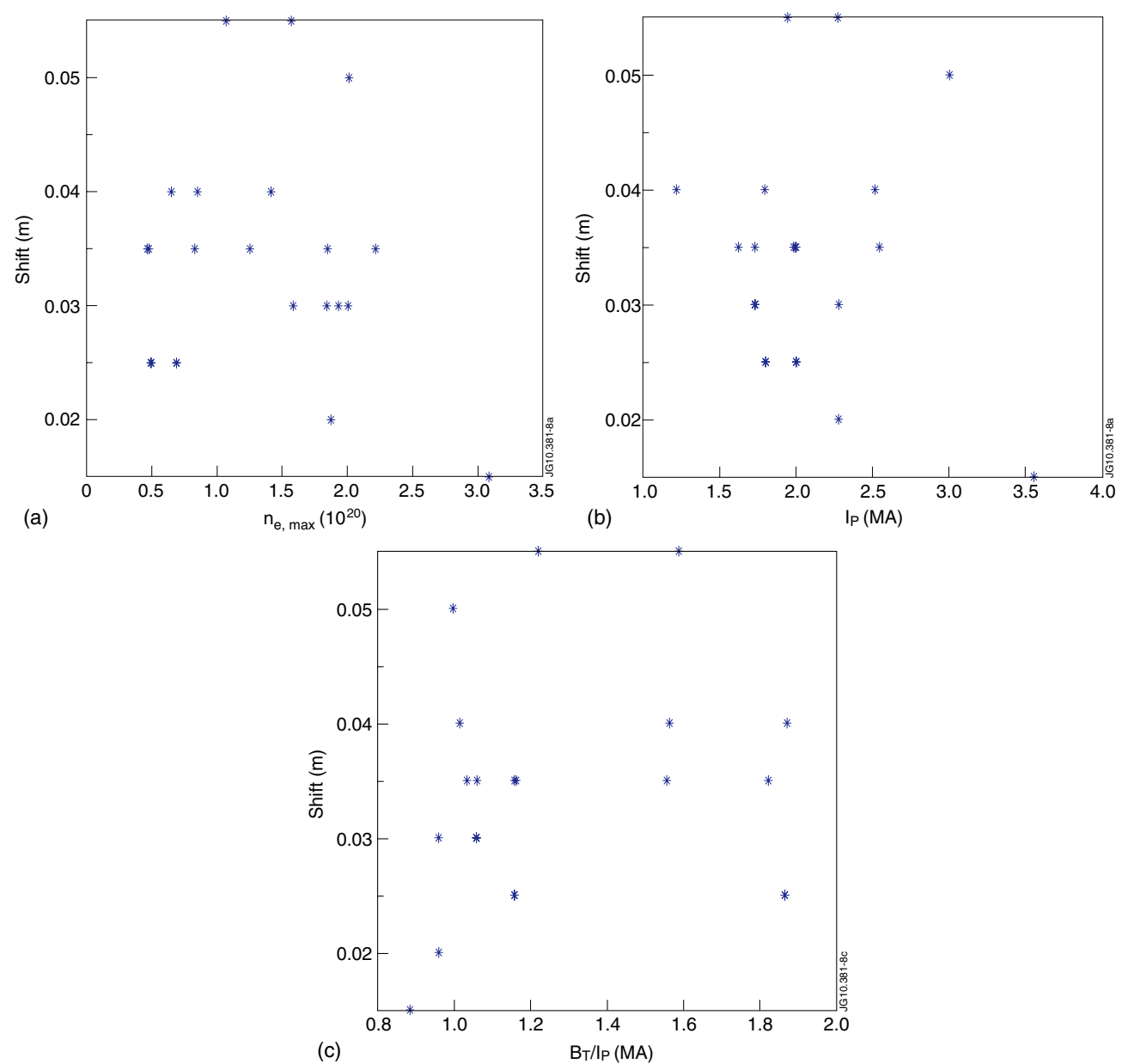

Figure 8. (a) The shift (m) versus the maximum line-averaged electron density measured by LIDAR Thomson scattering in units of $10^{20} \mathrm{~m}^{-3}$. (See table 2 for the plasma parameters of the shots considered.) (b) The shift (m) versus the plasma current $I_{P}$ (MA). (c) The shift versus the ratio $B_{T} / I_{P}$ (toroidal magnetic field/plasma current).

The code XLOC [7] is a simplified equilibrium code which is used for the determination of the X-point location and strike points of the open field lines derived from the X-point on the divertor tiles.

The result obtained after this comparison is that EFIT predicts a position of the lower outer strike point systematically in excess of $0.06 \mathrm{~m}$ with respect to XLOC calculations, confirming the shift in the same direction detected using the polarimetry analysis.

\section{Theoretical analysis of the coupling between Faraday and Cotton-Mouton effects and application to study shots at high density and high plasma current}

In section 1 the mutual interaction between Faraday rotation and the Cotton-Mouton was discussed in the context of the type II approximation.

Moving to a more general analysis, we start from the Stokes equations (2.2) to derive equations where the coupling terms between Faraday and Cotton-Mouton can be clearly identified. 
Defining

$$
F=\frac{s_{1}}{s_{2}}=\frac{1}{\tan 2 \psi} \quad \text { and } \quad C=\frac{s_{3}}{s_{2}}=\tan \varphi
$$

from the Stokes equations (2.2) the following system can be derived:

$$
\begin{aligned}
& \frac{\mathrm{d} F}{\mathrm{~d} Z}=-\Omega_{3}-\Omega_{3} F^{2}+\Omega_{1} F C+\Omega_{2} C, \\
& \frac{\mathrm{d} C}{\mathrm{~d} Z}=\Omega_{1}+\Omega_{1} C^{2}-\Omega_{3} F C-\Omega_{2} F .
\end{aligned}
$$

System (5.1)-(5.2), which is exactly equivalent to the Stokes model, can be considered as a generalized Volterra-like problem, with non-constant coefficients. The propagation of polarization in a plasma (without absorption) can be described using a first order nonlinear differential system in two variables: plasma polarimetry is intrinsically described by a bidimensional dynamical system, so chaotic behaviour cannot be realized [11].

The type II approximation (equation (2.12)) is obtained neglecting the terms $\Omega_{1} F C+\Omega_{2} C$ in equation (5.1) and the terms $\Omega_{1} C^{2}-\Omega_{2} F$ in equation (5.2).

It appears from the inspection of system (5.1)-(5.2) that two types of non-linearities appear in the propagation of polarization: (i) quadratic terms in $F$ and $C$; (ii) coupling terms $F^{*} C$. Since usually $\Omega_{3} \geqslant \Omega_{1}$ it could be expected that the main non-linearity in Faraday is due to the quadratic term $F^{2}$, while for Cotton-Mouton the main non-linearity would be the coupling term $F^{*} C$.

The magnitude of the terms at the right-hand side of (5.1)-(5.2) can be estimated solving directly the Stokes equations. Figures $8(a)$ and $(b)$ show how the non-linear terms play in the determination of the Cotton-Mouton effect: the values of $\mathrm{d} C / \mathrm{d} Z$ and $\Omega_{1}$ are plotted together versus the normalized coordinate along the beam path for channel 3 (figure $9(a)$ ) and channel 4(figure $9(b)$ ) at the time $t=18 \mathrm{~s}$, for the high density shot \#67777: it appears that the nonlinear terms become important for $Z>0$, in fact the terms in $C^{2}$ and $F^{*} C$ increase for $Z>0$ (i.e. in the upper part of the poloidal plane), their sum corresponding to $25 \%$ of the contribution to $\mathrm{d} C / \mathrm{d} Z$. Figures $10(a)$ and $(b)$ show a similar plot for the Faraday rotation angle, the values of $\mathrm{d} F / \mathrm{d} Z$ and $\Omega_{3}$ are plotted together for shot \#67777: it appears that the non-linear terms are negligible for channel 3 (figure $10(a)$ ), while the non-linear terms are important for channel 4 (figure $10(b)$ ), in this case, the Faraday $Z$-derivative is about eight times that for channel 3 and the term in $F^{2}$ is close to $80 \%$ of the value of $\mathrm{d} F / \mathrm{d} Z$.

It has been verified therefore that (for shot \#67777) the most important non-linear term for Faraday is the term $\Omega_{3} F^{2}$, while for Cotton-Mouton it is the term $\Omega_{3} F C$, in equation (5.1)-(5.2).

The importance of non-linear terms for Faraday rotation is confirmed by a comparison between the Faraday rotation measurements and the calculations for a high density/high current shot (\#75238) as shown in figure 11, where the experimental data (continuous line) are shown together with the numerical calculation (dashed line) and the linear formula (crosses): the value of $W_{3}$ slightly underestimates the Faraday measurement for the high current shot, while the numerical solution is in agreement with the measured Faraday rotation.

\section{Remarks on the mathematical forms of polarimetry constraints inside the equilibrium codes}

The previous sections contain information on the correct mathematical form to be used inside the equilibrium codes for the polarimetry constraints, for JET discharges. 

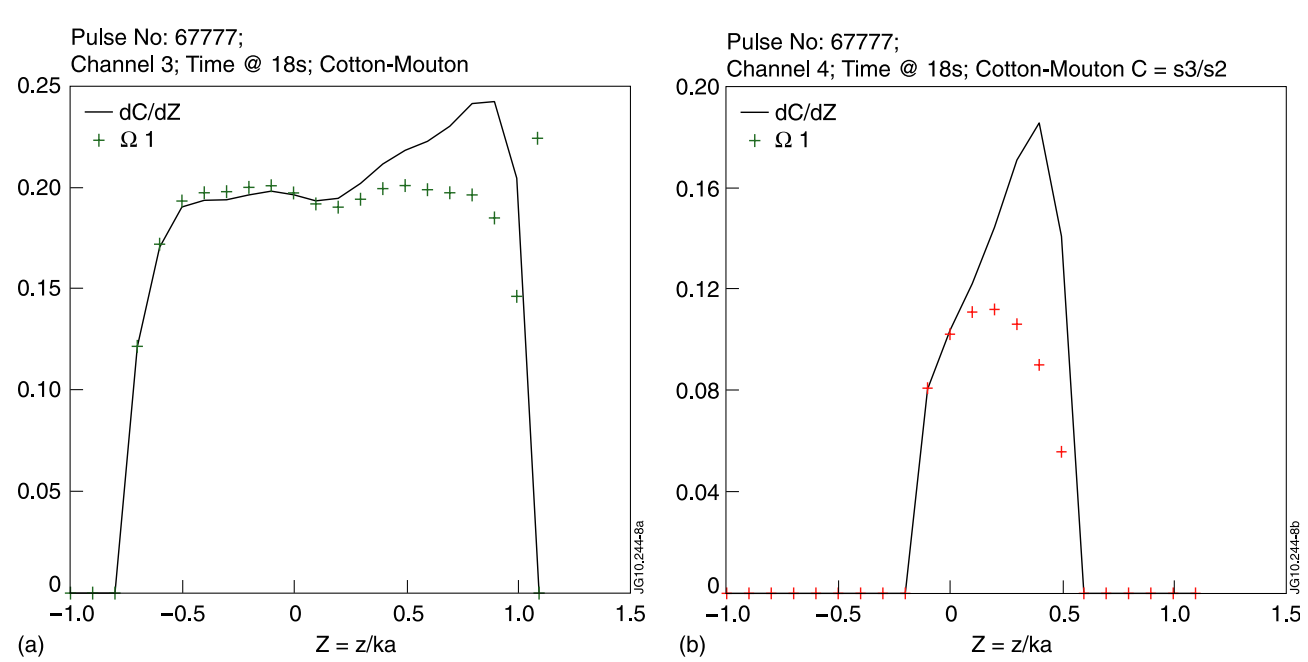

Figure 9. Cotton-Mouton. The left member of equation (5.2) (continuous line), and the values ('cross' symbols) of $\Omega_{1}$ are shown, for shot $\# 67777$, channel 3 (figure $9(a)$ ) and channel 4 (figure $9(b)$ ).
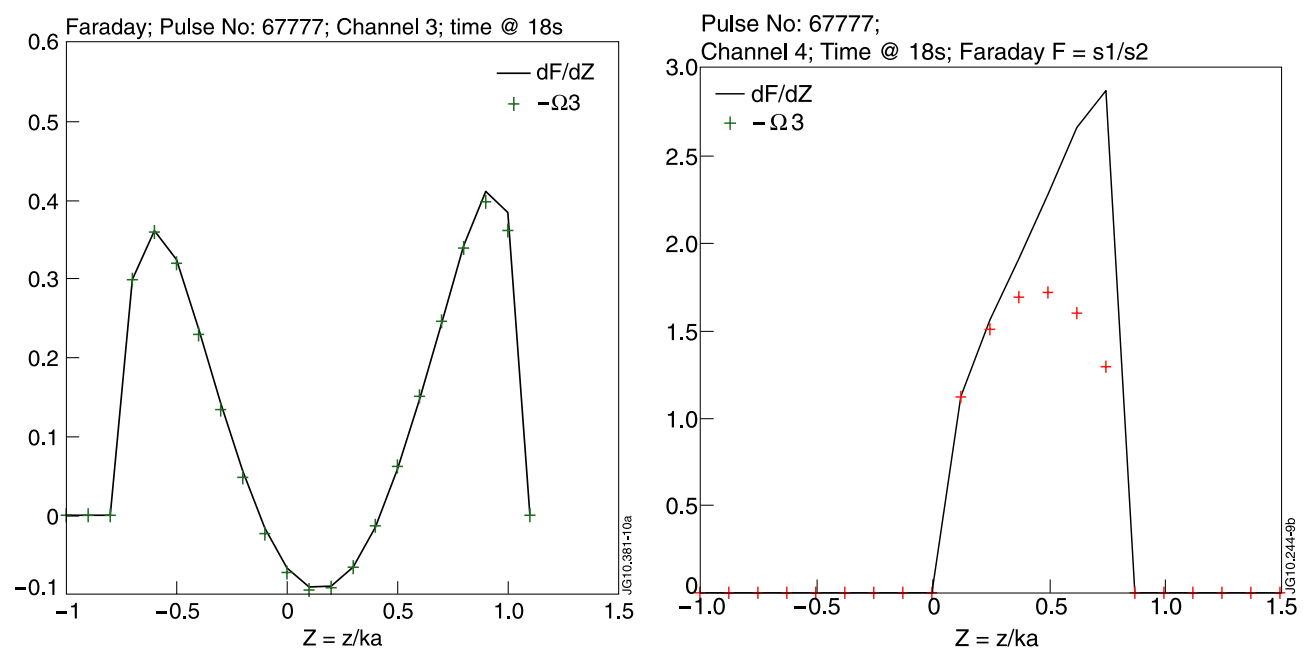

Figure 10. Faraday rotation. The left member of equation (5.1) (continuous line), and ('cross' symbols) the value of $-\Omega_{3}$ are shown, for shot $\# 67777$, time $=18 \mathrm{~s}$, channel $3(a)$ and channel $4(b)$.

In general, the constraint on Faraday rotation cannot be expressed using the 'linear form' (2.7), as it is used currently: the example of the calculations related to chord \#4 for shot \#67777 shows that the numerical model or type II is more adequate to describe the Faraday rotation at high density, and for shot \#75238, at high current and density.

The introduction of Cotton-Mouton as a constraint in the equilibrium code for an improved determination of plasma density must be treated carefully, since for CottonMouton the 'linear form' can be used only at low-medium density, while at high density the effect of Faraday rotation must be included. The simplest form of a model for CottonMouton, which includes the dependence upon Faraday rotation is represented by the type II approximation. 


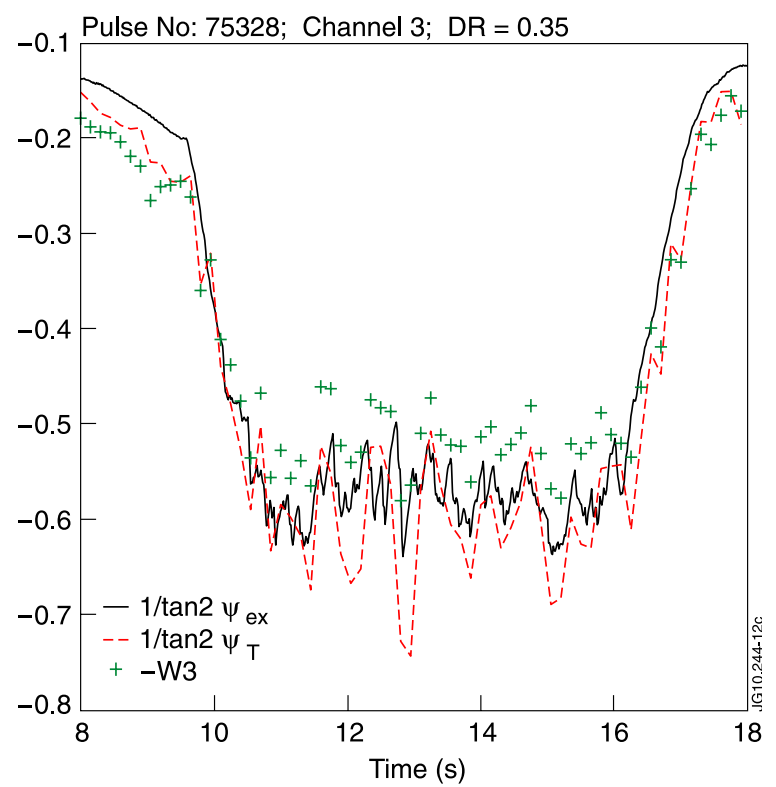

Figure 11. Faraday rotation measurement (continuous line, shot \#75238, channel 3) is plotted together with the calculated values (dashed line) using the solution of Stokes equations. The calculated values of $-\mathrm{W}_{3}$ (crosses) are shown

\section{Conclusions}

This paper presents a detailed analysis of Faraday measurements at JET, a comparison with available models and a rigorous approach to the mutual interaction between Faraday and Cotton-Mouton. The Faraday rotation can be calculated from the Stokes equations and the comparison between the calculations and the measurements can lead to information related to corrections of the position of the magnetic surfaces. Moreover, it turns out that the Faraday rotation cannot, in general, be represented by the linear expression of the type I approximation (expression (2.7), section 2). The Cotton-Mouton, at high density and current, must be calculated including the dependence from the Faraday rotation angle. This paper suggests that a test of the new mathematical forms of Faraday and Cotton-Mouton in the constraints of the EFIT equilibrium code is necessary to obtain accurate equilibrium reconstructions in all JET regimes of operations.

\section{Acknowledgments}

The authors would like to thank S Segre for interesting observations and suggestions about the analysis presented in this paper. They thank L Appel, M Brix, V Drozdov and E Solano for discussions about the polarimetry constraints in the EFIT equilibrium code. They also thank B Alper for the contributions on analysis of soft-X measurements.

This work, supported by the Euratom Communities under the contract of Association between EURATOM/ENEA, was carried out within the framework of the European Fusion Development Agreement. The views and opinions expressed herein do not necessarily reflect those of the European Commission.

(C) Euratom 2011. 


\section{References}

[1] Braithwaite G, Gottardi N, Magyar G, O’Rourke J, Ryan J and Veron D 1989 Rev. Sci. Instrum. 602825 Veron D 1974 Opt. Commun. 1095

[2] Orsitto F P, Boboc A, Mazzotta C, Giovannozzi E, Zabeo L and JET EFDA Contributors 2008 Plasma Phys. Control. Fusion $\mathbf{5 0} 115009$

[3] O’Brien D et al 1992 Nucl. Fusion 321351

[4] Guenther K et al 2004 Plasma Phys. Control. Fusion 461423

[5] Segre S E and Zanza V 2006 Plasma Phys. Control. Fusion 48339

[6] Solano E, private communication

[7] Sartori F, Cenedese A and Milani F 2003 Fusion Eng. Des. 66-68 735-9

[8] Segre S E 2003 J. Phys. D: Appl. Phys. 362806 (equations (48) and (49))

[9] Devaux S 2010 Talk given at JET Data Validation Coordination Meeting (27 July 2010, Culham Science Centre, Abingdon, UK)

[10] Alper Bprivate communication

[11] Glendinning P 2001 By the Peixoto theorem the dynamics of autonomous differential equations in a plane are relatively simple, there is no possibility for chaotic behaviour Stability, Instability and Chaos (Cambridge: Cambridge University Press) chapter 5

[12] Brix M, Hawkes N C, Boboc A, Drozdov V, Sharapov S and JET-EFDA Contributors 2008 Rev. Sci. Instrum. 79 10F325

[13] De Angelis R et al IAEA FEC 2010-Determination of $q$ profiles in JET by Consistency of Motional Stark Effect and MHD Mode location EXS/P2-03 